\title{
Observations of Coronal Mass Ejections from the Solar Mass Ejection Imager and Space Weather Implications
}

\author{
Janet C. Johnston ${ }^{1}$, David F. Webb ${ }^{2}$, Ghee Fry ${ }^{3}$, Joel B. Mozer ${ }^{1}$, \\ Thomas A. Kuchar ${ }^{2}$, Donald R. Mizuno ${ }^{2}$, and Timothy A. Howard \\ ${ }^{1}$ Air Force Research Laboratory, Space Vehicles Directorate, Hanscom AFB, \\ MA 01731-3010, USA \\ email: janet.johnston@hanscom.af.mil, joel.mozer@hanscom.af.mil \\ ${ }^{2}$ Institute for Scientific Research, Boston College, Chestnut Hill, MA 02467, USA \\ email: david.webb@hanscom.af.mil, thomas.kuchar@hanscom.af.mil, \\ donald.mizuno@hanscom.af.mil \\ ${ }^{3}$ Exploration Physics International, Inc. 6275 University Dr. NW, Suite 37-105, Huntsville, AL \\ 35806-1776, USA \\ email: gfry@expi.com \\ ${ }^{4}$ Physics Department, Montana State University, Bozeman, MT 59717, USA \\ email: thoward@mithra.physics.montana.edu
}

\begin{abstract}
The Solar Mass Ejection Imager (SMEI) was launched into a Sun-synchronous orbit in January 2003. Its mission objective is to detect and track coronal mass ejections (CMEs) from the Sun in order to improve space weather forecasts. In the three years since launch, over 200 CMEs, about 30 of which were Earth-directed, have been observed by SMEI. We have been able to track several of these CMEs from the SOHO LASCO coronagraphs $\left(<8^{\circ}\right.$ from the Sun) through the SMEI field of view $\left(>20^{\circ}\right)$ out to $0.5 \mathrm{AU}$ and beyond, and to observe the morphology and evolution of distinctive features over this wide distance range. We report on comparisons of measurements of CME parameters made in the inner heliosphere with the more typical measurements made nearer the Sun with coronagraphs. We illustrate SMEI's capabilities and present key statistical results on basic CME parameters and the use of SMEI-type data in space weather forecasting models. For example, timely observations by SMEI of CMEs en route to Earth could be input to DoD's operational Hakamada-Akasofu-Fry solar wind model to correct or refine its real-time forecasts of approaching disturbances.
\end{abstract}

Keywords. space vehicles: instruments, Sun: Coronal Mass Ejections (CMEs), (Sun:) solarterrestrial relations, interplanetary medium

\section{Introduction: SMEI Mission and Background}

The Solar Mass Ejection Imager (SMEI) is a collaborative project of the U.S. Air Force Research Laboratory, the University of Birmingham (UK), NASA, the University of California - San Diego, and Boston College. Its primary mission is the detection and tracking of coronal mass ejections (CMEs) from the Sun to Earth to improve space weather forecasts (Jackson et al. 2004). It was launched 6 January 2003 on the Coriolis spacecraft and has a design mission lifetime of 3-5 years. The SMEI instrument has three camera/baffle systems, each having a $30 \times 60^{\circ}$ field of view (FoV) resulting in a total field fan survey $155^{\circ}$ wide. SMEI images faint CMEs to a level less than $1 \%$ of the background (visible) sky brightness. 
a

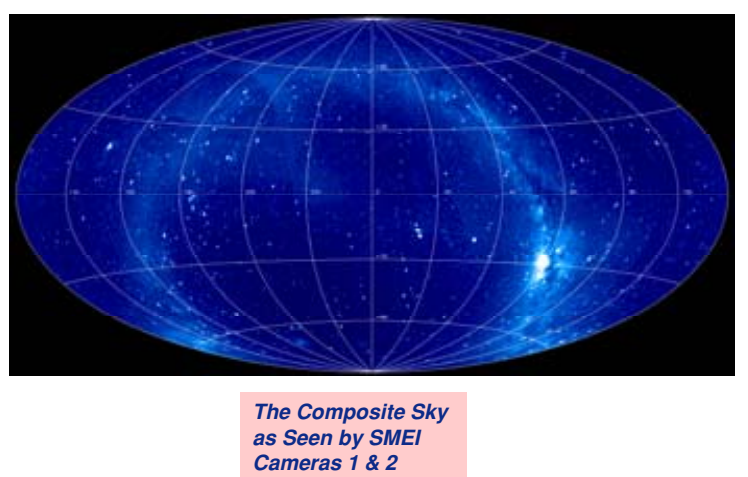

b

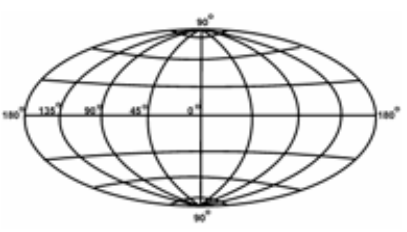

Figure 1. (a) Composite SMEI all-sky map, made using two of the three SMEI cameras.

(b) Hammer-Aitoff map projection.

SMEI has been operating in "normal" observing mode approximately $85 \%$ of the time; it obtains a full sky image every orbit ( $\sim 100$ minutes). Figure 1a is a composite allsky image made using only two cameras. Figure $1 \mathrm{~b}$ shows a graphic of the projection used (Hammer-Aitoff). Data latency for quicklook images is less than 8 hours. SMEI has successfully satisfied its mission objective, observing over 200 CMEs in three years of operation, including at least 30 geoeffective CMEs.

\section{Performance and CME Statistics}

We have completed a detailed survey of the first 1.5 years of SMEI data (Webb et al. 2006). SMEI observed 139 CMEs in this period. The estimated observation rate is $0.3 \mathrm{CMEs} /$ day, compared with LASCO's rate of $>3 \mathrm{CMEs} /$ day. The mean duration was $16.3 \mathrm{hr}$ with a range of $3.5-70 \mathrm{hr}$. Mean angular speeds were measured to be $1.1^{\circ} / \mathrm{hr}$. Using a propagation model (P-approximation; Howard et al. (2006)) yields a mean speed of $473 \mathrm{~km} / \mathrm{sec}$ with a range $51-1611 \mathrm{~km} / \mathrm{sec}$. For comparison, the LASCO mean CME speed was $507 \mathrm{~km} / \mathrm{sec}$ and the Helios-2 (Webb \& Jackson, 1990) mean CME speed was $\sim 500 \mathrm{~km} / \mathrm{sec}(1976-1979)$. During this period SMEI detected $\sim 20$ halo CMEs at $\sim 1 / 3$ of Sun to Earth distance, thus demonstrating the capability to detect CMEs 10 hours to over 24 hours before Earth arrival.

The discrepancy in the number of events detected by LASCO and by SMEI may have several explanations, such as obscured regions in the FoV via particle contamination, shuttering, or auroras, or the fading with height of CMEs, backsided CMEs that are too distant from SMEI, or LASCO multiple-event episodes evolving into single SMEI features. This is a subject of ongoing research.

\section{Examples: CME Morphology}

Figure 2 shows an example of point-for-point tracking of distinctive features from the LASCO FoV into SMEI's FoV for an event in February 2003. Figure 2 shows am elongation-time plot of a wedge-shaped feature, yielding a speed of $610 \mathrm{~km} / \mathrm{sec}$. Also, for this event, the angular span of the wedge is measured throughout the track and remains approximately constant. In this case an estimate of the source location and launch time can be made to minimize projection effects in the computed speeds. Deceleration of the feature by the time it traverses the SMEI FoV is not observed or is minimal. 

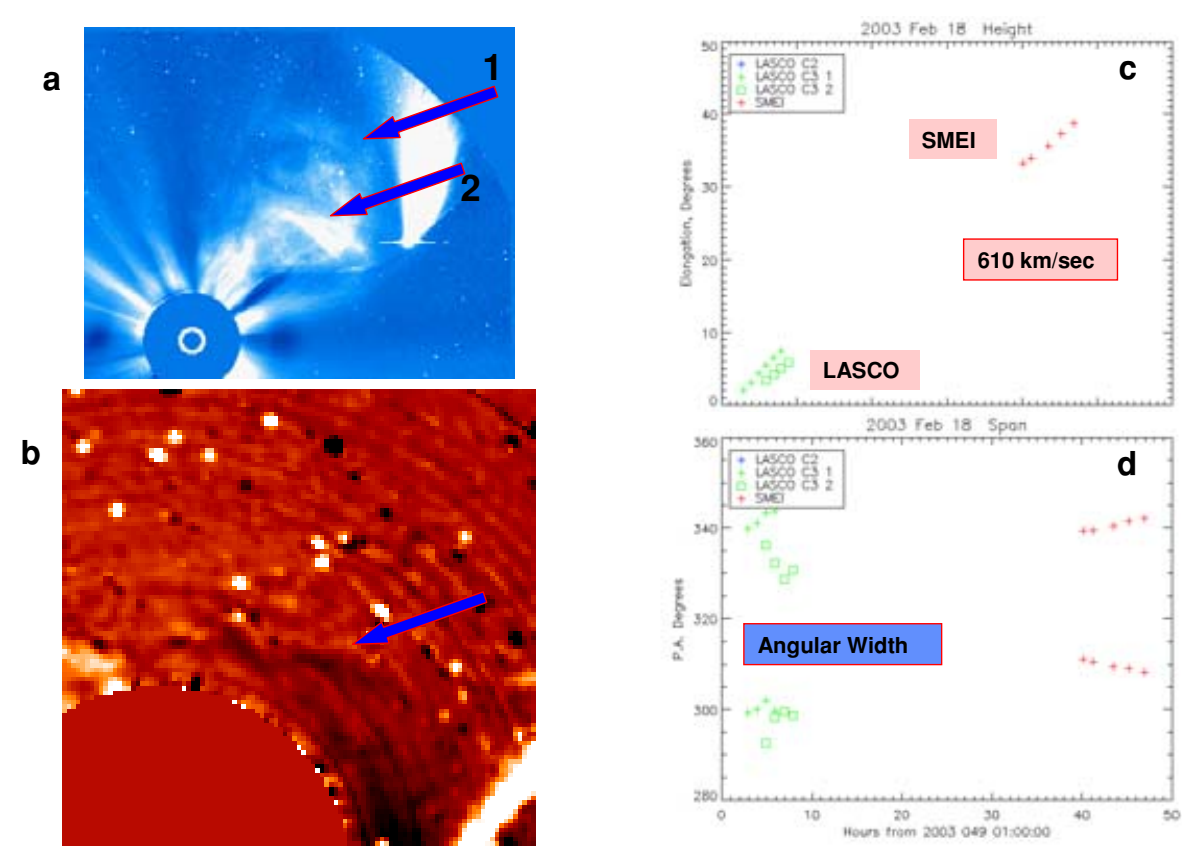

Figure 2. Tracking a wedge-shaped feature in LASCO (a) and SMEI (b). The leading edges of the features are used in the elongation/time plots (c) and for angular span measurements (d). SMEI images are running orbit differences.

\section{Space Weather Forecasting}

CMEs are a primary cause of severe space weather at Earth because they trigger geomagnetic storms that can damage space and ground-based assets. SMEI's primary purpose is to demonstrate the ability to track CMEs from near the Sun to Earth, thus providing a new capability for forecasting storms. SMEI has accomplished this objective by detecting geoeffective CMEs at elongations of $20-30^{\circ}$, equivalent to $\sim \frac{1}{3}$ of the distance from the Sun to Earth. Depending on the speed of the CME front, these distances correspond to advance-warning times of 10 hours to 2 days.

We performed several studies of SMEI's capability to detect and track geoeffective CMEs. In one study (Webb et al. 2006), we separately examined the sources of moderate (peak Dst $<-60 \mathrm{nT}$ ) and intense (peak Dst $<-100 \mathrm{nT}$ ) geomagnetic storms during 2year intervals. SMEI observed associated Earthward-directed CMEs for 85\% (39 of 46) and $87.5 \%$ (14 of 16) of these storms, respectively. For the intense storms, the mean lags between the first SMEI observation of the CME and 1) Earth arrival of the associated shock and 2) onset of the storm were 18.6 and 29.25 hours, respectively. In another study (Howard et al. 2006), we used distance-time plots of LASCO halo CMEs that were associated both with SMEI transients and shocks detected by the ACE spacecraft near 1 AU to compare the predicted and actual arrival times. The mean range between these values was about $10 \mathrm{hr}$., representing an approximate $30 \%$ improvement in the accuracy of predicting CME arrival times compared to published results using LASCO data only. Our main conclusion is that SMEI could detect the CMEs causing most major geomagnetic storms, from half a day to 2 days before storm onset and, therefore, that an operational version of a SMEI-type instrument could provide improved early warning of storms. 
A separate study used the Hakamada-Akasofu-Fry (HAF; Hakamada \& Akasofu, 1982; Fry et al. (2001)) solar wind model that provides real-time forecasts of CME position and interplanetary shock arrival time (SAT) at Earth. Howard et al. (2006) studied 20 CMEs observed by SMEI that were associated with geomagnetic storms. They computed distance-time profiles to determine CME speed and to predict the shock arrival time at Earth. For the 20 observed CMEs HAFv.2 was used to make 13 correct predictions: 9 shocks and 4 no-shocks (correct nulls). SMEI observations alone led to 14 correct predictions: the 9 HAF shocks and 5 additional shocks. Thus, near real-time SMEI observations would have increased the HAF correct forecast rate by $38 \%(5 / 13)$.

\section{Conclusions}

SMEI has successfully demonstrated the capability of an all-sky white light imager to detect and track CMEs. Improvements to space weather forecasting using SMEI data are currently being explored and quantified.

We have shown that it is possible to track structures, point for point, from LASCO into the SMEI FoV, at least in simple cases of limb events. The evolution of distinctive features entering the gap region between LASCO and SMEI is less certain for more complicated or multiple events. However, we are in the process of a detailed comparative study of LASCO-SMEI events. Such long-distance range CME tracking ( $20^{\circ}$ to $>1$ A.U.) holds potential for understanding the physical processes of CME motion in the heliosphere.

The STEREO mission due to be launched later this year may overlap with SMEI observations, with SMEI providing context information for heliospheric structures viewed by the STEREO imaging instruments. For more information on the Solar Mass Ejection Imager visit http://smei.nso.edu/.

\section{References}

Fry, C.D., W. Sun, C.S. Deehr, M. Dryer, Z. Smith, S.-I. Akasofu, M. Tokumaru \& M. Kojima 2001, J. Geophys. Res. 106, 20985

Hakamada, K. \& S.-I. Akasofu 1982, Space Sci. Rev. 31, 3

Howard, T.A., D.F. Webb, S.J. Tappin, D.R. Mizuno \& J.C. Johnston 2006, J. Geophys. Res. 111, A04105, doi:10.1029/2005JA011349

Jackson, A. Buffington, P. P. Hick, R.C. Altrock, S. Figueroa, P.E. Holladay, J.C. Johnston, S.W. Kahler, J.B. Mozer, S. Price, R.R. Radick, R. Sagalyn, D. Sinclair, G.M. Simnett, C.J. Eyles, M.P. Cooke, S.J. Tappin, T. Kuchar, D. Mizuno, D.F. Webb, P.A. Anderson, S.L. Keil, R.E. Gold \& N.R. Waltham 2004, Solar Phys. 225, 177

Webb, D.F. and B.J. Jackson 1990, J. Geophys. Res. 95, 20641

Webb, D.F., D. R. Mizuno, A. Buffington, M.P. Cooke, C.J. Eyles, C.D. Fry, L.C. Gentile, P.P. Hick, P.E. Holladay, T.A. Howard, J.G. Hewitt, B.V. Jackson, J. C. Johnston, T. A. Kuchar, J. B. Mozer, S. Price, R. R. Radick, G. M. Simnett, and S. J. Tappin 2006, J. Geophys. Res. (submitted) 